\title{
Testing for Phenotypic Plasticity
}

\author{
Aja Watkins*
}

\begin{abstract}
Phenotypic plasticity, or an organism's capacity to change its phenotype in response to environmental variation, is a pervasive - perhaps even ubiquitous-feature of the biological world. Accordingly, plasticity research suggests serious implications for biological theory, including evolutionary theory. The theoretical implications of plasticity have growing support from empirical literature documenting the range, extent, and adaptiveness of plasticity. However, the empirical evidence for particular instances of plasticity has still not been adequately scrutinized by biologists or philosophers. After reviewing some important conceptual and theoretical background information, this paper synthesizes some past discussions of the methodology of plasticity studies and provides some novel methodological guidance for plasticity researchers. My conclusion is that not all plasticity studies should carry the same weight: there are patterns we can identify in the features of such studies that should contribute to (or detract from) their credibility. In particular, I argue that some types of organisms and some types of plastic phenotypes are more conducive to the empirical study of plasticity than others.
\end{abstract}

\section{Keywords}

phenotypic plasticity $\bullet$ developmental plasticity $\bullet$ adaptive plasticity $\bullet$ ANOVA $\bullet$ reaction norm

\section{Introduction}

Phenotypic plasticity is the responsiveness of an organism's phenotype to environmental variation. Construed broadly, phenotypic plasticity is ubiquitous. Some well-studied examples include environment-dependent variation of: color in butterflies (e.g., Nijhout 1991), horn growth in male dung beetles (e.g., Emlen and Nijhout 1999), defensive chemicals emitted by plants (e.g., Heil 2010), caste membership in social insects (e.g., Kamakura 2011), sex determination in many reptiles (e.g., Bull 1980), hatching times in tadpoles (e.g., Warkentin 2005). However, plasticity can include all forms of behavioral responsiveness, including responsiveness to signals and learning.

*Philosophy Department, Boston University, Boston, MA, USA, ajawatki@bu.edu (iD) https://orcid.org/0000-0003-0171-8738

Received 17 June 2020; Revised 27 November 2020; Accepted 27 November 2020 doi:10.3998/ptpbio.16039257.0013.003 
Many have claimed that the implications of plasticity research are wide-reaching. For example, phenotypic plasticity has implications for evolutionary biology. Gould and Lewontin $(1979,592)$ classified phenotypic plasticity as a type of "adaptation without selection": the fact that phenotypic variation may not require genetic variation means that selective environments can increase the propensity of a phenotype in a population without requiring genetic mutation or reproduction. Selection can even act on the propensity to respond to an environmental cue, thereby increasing or decreasing the plasticity exhibited by an entire population. Philosophers and biologists have recently become interested in whether such alternatives to standard-issue adaptation by natural selection should support replacing the Modern Synthesis with an Extended Evolutionary Synthesis (e.g., Pigliucci 2007; Gilbert and Epel 2015, 436). The debate hinges in part on whether or not we can actually distinguish adaptation via plasticity from adaptation via selection on genes, as Gould and Lewontin seem to suggest we can (see Wray et al. 2014 and Laland et al. 2014 for an overview of this debate).

Phenotypic plasticity has, additionally, been used to argue that the "nature versus nurture" debate is ill-conceived, because the phenotype that results from plasticity is inextricably linked to both an organism's genome and its environment (e.g., Keller 2010). Lewens (2010), for example, notes that if we admit that outcomes for a given genome always differ depending on environmental factors, then we can't use whether an outcome was caused by genetic or environmental factors to demarcate the distinction between innate or social differences. Pigliucci (2001) similarly concludes on the basis of plasticity research that "scientists cannot and should not venture to say ... that we understand the interactions of genes and environments in humans to a point that that knowledge can safely be used to inform social policies" (261). These implications of phenotypic plasticity research are grounded in empirical research on specific instances of plasticity.

The aim of this paper is to address some of the long-standing issues pertaining to the methodology of plasticity research. Some of this paper reviews and synthesizes what other researchers have said about the methodology of plasticity studies, a synthesis which is hopefully helpful as a reference. Then, I raise some novel concerns. In particular, I will argue that there are patterns in the ways in which various experimental setups in plasticity studies lend more or less support to the conclusions drawn.

The structure of the paper is as follows. First, in section 2, I will discuss how researchers collect evidence for the hypothesis that plasticity, rather than genetic variation, is causing some particular phenotypic variation. As we will see, this is easier in some cases than others: use of genetically identical organisms (clones) and/or examination of reversible plasticity will result in more credible scientific results. Next, in section 3, I will look at how adaptive phenotypic plasticity can be tested for; in this case, use of irreversible plasticity becomes important. I will also distinguish between adaptive plasticity and plasticity that emerged as an adaptation, and examine some studies that purport to show the latter.

\section{Phenotypic Plasticity}

In this section, I will first clarify what phenotypic plasticity means, as well as how we quantify it. Then, I will discuss various methodological issues with testing for the presence of phenotypic plasticity. 


\subsection{Defining and Quantifying Phenotypic Plasticity}

Mary Jane West-Eberhard (2003), who has inarguably been very influential to the field of plasticity research, defines phenotypic plasticity (or, "plasticity," for short) as: "the ability of an organism to react to an environmental input with a change in form, state, movement, or rate of activity" (34). She clarifies this definition in several ways.

First, the definition is not about a single genotype responding to the environment, but about the organism doing so, because "the phenotype of an individual exists as a parentally provided structure before its own genotype begins to act" (34-35). For instance, gametes, which do not contain a complete genome, have phenotypes and can be plastic prior to fertilization. WestEberhard does not want to limit plasticity research only to organisms that have a complete genotype (admittedly, it is also unusual to allow gametes to count as organisms). I will discuss this part of West-Eberhard's definition several more times below.

Second, plasticity can be either adaptive or nonadaptive. West-Eberhard wants to include nonadaptive - even maladaptive-plasticity in the definition because, she says, we cannot always tell which traits are adaptive or not, and some traits which are not adaptive may become so. For example, poison dart frogs, whose toxicity is solely the result of their diet, likely did not initially benefit from self-intoxicating, prior to having developed a defense (e.g., Tarvin et al. 2017). Adaptive plasticity will be discussed at greater length in section 3 .

Third, there are several other distinctions West-Eberhard does not wish us to include in the definition: active versus passive, "noise," continuous versus discrete variation (35-36). The definition is thus intentionally broad. Anything that constitutes a change in "form, state, movement, or rate of activity" (these being parts of an organism's phenotype) as a reaction to environmental input counts as plasticity. Not surprisingly, West-Eberhard thinks plasticity is a pervasive part of the biological world.

I agree with West-Eberhard's definition of phenotypic plasticity, given her arguments in favor of it, but others may object that it is too broad. Technically, every trait of every organism is responsive to the environment, but there are some ways in which this responsiveness broadly construed seems trivial or at least uninteresting. A cut on my finger, or my height as a function of gravitational force, or my hair length are all, technically, instances of plasticity, by West-Eberhard's definition (although West-Eberhard herself never explores these extreme cases). One possible problem with such a broad definition is that we would no longer be motivated to test for the presence of phenotypic plasticity, if it is virtually guaranteed that any given trait is plastic. However, for phenotypic plasticity to have the theoretical implications that West-Eberhard argues that it has (such as providing a mechanism for speciation events, or problematizing the distinction between environmental and genetic causation), plasticity needs to be ubiquitous in this way. Because this paper is about the methodology of plasticity studies, rather than the theoretical implications of phenotypic plasticity, it will be outside the scope of the paper to show exactly how the ubiquity of plasticity leads to each of the theoretical implications West-Eberhard argues for. Suffice it to say that a broad definition of plasticity is a crucial part of these arguments, so I will continue to adopt the broad definition herein.

A second possible criticism of West-Eberhard's definition is that phenotypic plasticity, under her definition, is a property of individual organisms. We might not think that it is particularly useful or interesting to know if a single given organism has a plastic trait or not; rather, we probably are more interested in whether a category of organisms exhibits plasticity. For example,

1. West-Eberhard's use of the word "ability" in the definition may make plasticity sound active; she does not intend it this way.

๑ OPEN ACCESS - PTPBIO.ORG 
Pigliucci (2001)'s definition uses "genotype" instead of "organism" in this way (1). I The difference between these definitions-namely, in what exactly we take plasticity to be predicated of-will become important as we discuss how we measure plasticity.

One way to quantify phenotypic plasticity is to use analysis of variance (ANOVA). The idea behind ANOVA is to take a variety of genotypes and a variety of environments and assess the resulting phenotype for each genotype-environment pair. Because we are interested in measuring plasticity, we will be interested in knowing how much of the resulting phenotypic variation was due to environmental, rather than genetic, effects. However, Lewontin $(2006,521)$ has argued that ANOVA is unable to distinguish between genetic and environmental variation as the cause of phenotypic variation. First, ANOVA alone will not distinguish between amount of environmental variation and amount of organismal sensitivity to environmental variation, because the outcomes (and resulting averages) are measured in phenotype differences. Both a highly variable environment and an only slightly variable environment could produce the same variability in phenotype, depending on the organisms' sensitivity to environmental variation; the results of ANOVA only examine the variability of the phenotype, not the variation in the environment. Thus it cannot be said that the environmental variation caused the phenotypic variation. Second, ANOVA depends on the genotypes and environments sampled, making the resulting analysis necessarily localized and resistant to generalization. Lewontin (2006), and also Sober (1988a), therefore conclude that ANOVA cannot tell us how much effect genetic versus environmental differences had on the phenotype.

Lewontin (among others) recommends that, instead of ANOVA, we look at reaction norms. In practice, most researchers opt to use both ANOVA and reaction norms. Reaction norm graphs plot different phenotypes ( $y$-axis) against different environments ( $x$-axis) for each genotype (each line). Figure 1 gives a typical set of examples. (Note that phenotypes and environments may be discrete or on a spectrum. Genotypes must be discrete.)

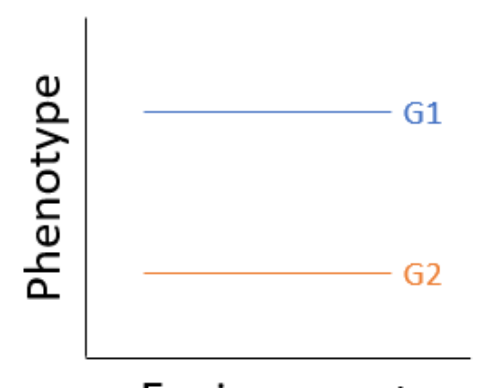

a. Environment

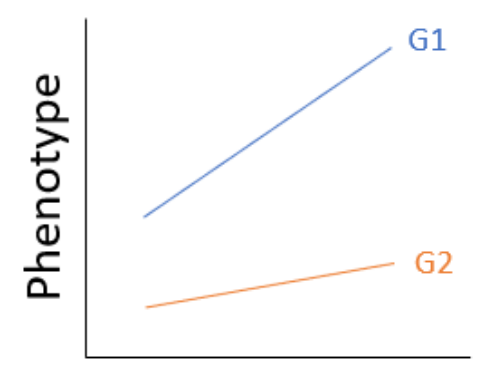

d. Environment

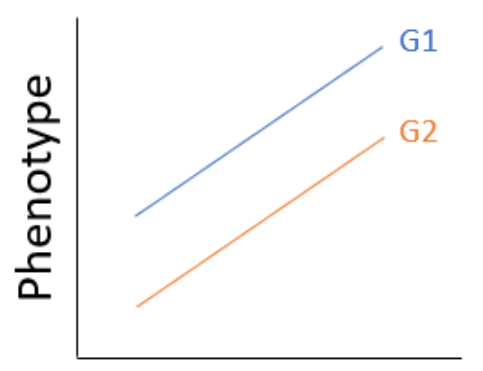

b. Environment

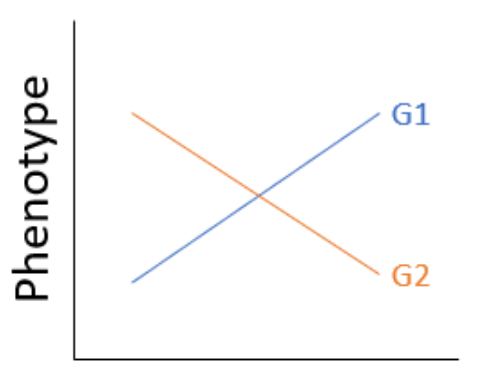

e. Environment

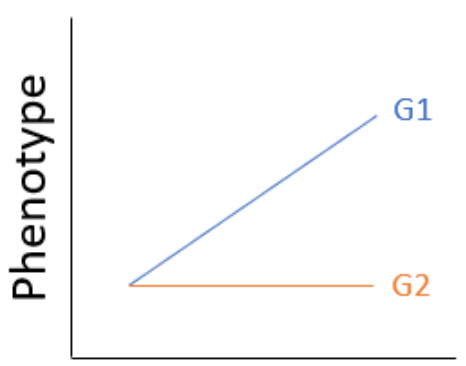

c. Environment

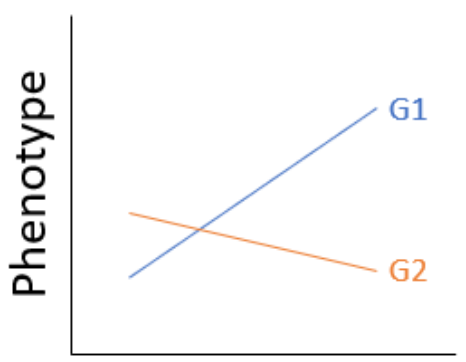

f. Environment

Figure 1: Example reaction norms. For a similar overview, see Pigliucci (2001, 6).

2. Note that Schlichting and Smith (2002)'s definition agrees with West-Eberhard's. 
Figure 1(a) shows two genotypes that differ from one another in phenotype, but do not change phenotype based on their environment; neither has any plasticity. Figure 1(b) shows two genotypes which both are equally plastic, but which still differ from each other within each environment. Like in Figure 1(a), the mean phenotypes of $G 1$ and $G 2$ are different. In figure 1(c), $G 1$ is plastic while $G 2$ is not. However, in one environment-where their reaction norms intersect, at the far left-G1 and $G 2$ display the same phenotype. It is only by observing the genotypes in multiple environments that we can tell that the two genotypes differ at all in resulting phenotype. In figures 1(d), (e), and (f), $G 1$ and $G 2$ are both plastic. In (d), $G 1$ is more plastic than $G 2$. In (e), $G 1$ and $G 2$ are equally plastic, but in different directions, and their mean phenotype is also the same. Here the genotypes in effect "switch" phenotypes when the environment changes. In (f), $G 1$ and $G 2$ also show plasticity in the opposite direction, but the amount of plasticity also differs, as does the phenotypic mean. ${ }^{3}$

Looking at reaction norms suggests a possible measure of how much plasticity is present in a given case: the (absolute value of the) slope of the line for each genotype. Thus $G 1$ has greater plasticity than $G 2$ in figures 1 (d) and (f), as evidenced by its steeper slope. The slope thus allows for precise comparison of the degree of plasticity exhibited by different individuals, or populations under particular conditions. (Of course, this will only work when the reaction norm is linear, which, across more than two environments, it frequently is not.) However, note that it does not enable comparisons of plasticity regarding different traits; the phenotype measure (on the $y$-axis) has to be the same for comparisons to be meaningful.

Using the slope as a measure of plasticity means that a genotype that had the same phenotype regardless of environment would have a line with a slope of 0 , and would also not be considered plastic. Some have objected that, in fact, we have evidence to believe that much consistency in phenotype is due to a sort of plasticity ${ }^{\mathbb{4}}$ For example, the maintenance of homeostasis requires a plastic response to varying environments (e.g., West-Eberhard 2003, 45). However, defenders of reaction norms would say the problem here is not with using slope to measure plasticity, nor with our definition of plasticity (which requires a change in phenotype). Rather, the problem is with our selection of which phenotype we measure. If we measure an endothermic animal's body temperature, it will appear to be relatively unchanging regardless of the environmental conditions. However, if we measure the same animal's metabolism and energy expenditure, these will vary, in order to produce an outcome that appears constant. So which phenotype we take to be a candidate for plasticity will matter to what we find; just because a particular phenotype measurement makes the phenotype seem constant across environments, does not entail that there is no plasticity occurring. By contrast, if a phenotype measurement does show change across environments, then that indicates there is likely plasticity occurring. While the nuances involved in selecting traits and environments across which to measure plasticity may be well-understood by researchers, it is important to point out that this slightly complicates the use of the slope as a measure of plasticity.

The reader may have noticed that, in switching to using reaction norms to measure phenotypic plasticity, it appears as though we are suddenly using a definition of phenotypic plasticity that requires it to be a property of genotypes, rather than individual organisms, because reaction norms (and their slopes) are properties of genotypes. I have already stated that I want to

3. In case it is not intuitive why phenotype "switching" would ever occur, here is an example: in the butterfly Bicyclus anynana, males and females alternatively display courtship behavior depending on their larval rearing temperatures (Prudic et al. 2011). Wet season females are more eager to lay eggs, and so more aggressively pursue mating opportunities.

4. In this spirit, McShea $(2012,664)$ says that "plasticity is the tendency for an entity to find a particular trajectory from a variety of different starting points." 
resist defining phenotypic plasticity as a property of genotypes, for all of the reasons that WestEberhard gives, even though hers is an unusual definition of plasticity in relation to the literature. However, I also agree with Lewontin that reaction norms are our best bet for measuring phenotypic plasticity. Unfortunately, this leads me to conclude that, when we are studying plasticity using reaction norms, we are not actually measuring plasticity per se; we are instead measuring the responsiveness of a genotype (or, a collection of genotypes) to change in the environment. Future work is needed to align studies which use reaction norms with the (theoretically necessary) definition of plasticity as a trait of organisms, not genotypes. In the remainder of this paper, I will disregard this discrepancy, and assume that reaction norms are able to directly measure plasticity.

\subsection{Testing for Phenotypic Plasticity}

One might imagine that a biologist who wished to study plasticity would be looking for evidence for the following hypothesis:

Naïve Plasticity Hypothesis: Observed phenotypic variation is the result of plasticity (rather than genetic variation).

I will revise this hypothesis below, but for now the basic idea of testing for phenotypic plasticity is to isolate phenotypic variation due to genetic variation from phenotypic variation due to variation in reactions to environmental cues. The former is not plasticity; the latter is.

Recall that some claim that even traits which show a flat reaction norm graph, such as traits that are the product of homeostasis, are the result of plasticity. Here is our first possible epistemic problem with testing for plasticity: it would appear there is an asymmetry between the Naïve Plasticity Hypothesis and the alternative hypothesis, which says there is no plasticity occurring (i.e., that all phenotypic variation is genetic variation). If we observe different phenotypes in different environments, the Naïve Plasticity Hypothesis is confirmed. However, if we observe the same phenotypes in different environments, this does not disconfirm the Naive Plasticity Hypothesis at all. Rather, we may have been measuring an inappropriate phenotype for the research context (like body temperature instead of metabolic rate). Additionally, we may have been observing an inappropriate set of environments; organisms may have no measurable phenotypic difference between Environment 1 and Environment 2, for instance, whereas had we exposed them to Environment 3 we would have measured phenotypic change and therefore plasticity. In at least these two cases, the Naïve Plasticity Hypothesis does not receive disconfirmation, despite no observation of plasticity.

The problem can be addressed by updating the Naïve Plasticity Hypothesis to what I will call the Relativized Plasticity Hypothesis, the one researchers are actually focused on:

Relativized Plasticity Hypothesis: Observed variation on phenotypes $P_{1}, P_{2}, \ldots, P_{m}$ across environments $E_{1}, E_{2}, \ldots, E_{n}$ is the result of plasticity (rather than genetic variation).

The Relativized Plasticity Hypothesis is, as the name would suggest, relativized to a specific set of phenotypes and environments. Note that this does not imply that plasticity is itself a property of traits; plasticity is still best conceived of as property of organisms, or at least of genotypes. Such a hypothesis could be disconfirmed without disconfirming the more general Naïve Plasticity Hypothesis. The Relativized Plasticity Hypothesis is already used by plasticity researchers rather than the Naïve one, albeit often implicitly. (I will refer to the Relativized Plasticity Hypothesis as simply the Plasticity Hypothesis from here on.)

๑ OPEN ACCESS - PTPBIO.ORG 
I will now make two more substantive points about the methodology of plasticity studies. In both cases, I will be showing that some experimental setups are better equipped to confirm the Plasticity Hypothesis than others.

The first point is about the fact that reaction norms are properties of genotypes. Of course, there is a relationship between genotypes and individual organisms; one could make a reaction norm where each line was a separate individual organism, and those lines would also (roughly) still correspond to individual genotypes. One obvious problem with doing this has to do with sample size: for statistical reasons, scientists need to be able to use multiple members of a population to establish that a reaction norm line looks a particular way. (Usually, it would not make for a very interesting study if we tested one deer's changes in behavior in the presence or absence of wolves; the conclusion would be something like "this particular deer shows phenotypic plasticity in response to predation.") However, using multiple organisms in order to increase sample size not only means we are no longer attributing plasticity to individual organisms, as West-Eberhard's definition would require, but also that we are not attributing plasticity to individual genotypes, insofar as different individual organisms often have different genotypes. Below, I will suggest that using organisms which are more genetically similar mitigates this problem, by degrees.

There is a second problem: for irreversible plasticity, not even the same population in different environments can be used. Instead, members of one larger population get isolated into different environmental conditions. The phenotypic measurement for each sub-population is presumed to be the phenotype that the other sub-population would have had if they had been placed in the same environmental condition. This presumption relies on a counterfactual-what phenotype some individuals would have had - that cannot be tested, for an individual or for a group. Notice that there is a sense in which that counterfactual cannot be tested in the case of reversible plasticity, either: namely, we can never check and see if any organism "would have done otherwise" at a slightly altered environment at the same time; all experiments with reversible plasticity will involve measuring organisms' phenotypes at different times. Specifically, organisms are not expected to respond identically to multiple, repeated exposures to the same stimulus, because of the effects of learning or priming. However, our ability to test the counterfactual claim about what phenotype would have been exhibited is even worse in the case of irreversible plasticity than it is in the case of reversible plasticity. I do not find researchers affording studies of irreversible plasticity less credence than studies of reversible plasticity, although my argument is that maybe they should.

Figures 2 and 3 give some examples (based on real studies) of how reaction norms using multiple organisms are formed. In figure 2, a single reaction norm line is drawn between the average phenotype of all individual organisms exposed to each environment. In figure 3, several reaction norm lines are drawn between (genetically) similar organisms in each environmental condition. The important thing here is that these organisms are genetically beterogeneous (even if they are siblings, or otherwise similar). The effect of genetic variation on phenotypic variation has not been controlled for. Additionally, reaction norms are supposed to be properties of single genotypes (as in figure 11). An entire population, even of genetically similar organisms, cannot be properly said to have its own reaction norm.

The situation for irreversible phenotypes can be improved slightly in cases where the same genotype, if not the same organism, is used. Some asexually reproducing organisms (or otherwise cloned organisms) can come close to accomplishing this. There will be less genetic variation in a population of clones than in a population of conspecifics or even siblings in a sexually reproducing species. This does a better job of approximating the idea of one reaction norm for 


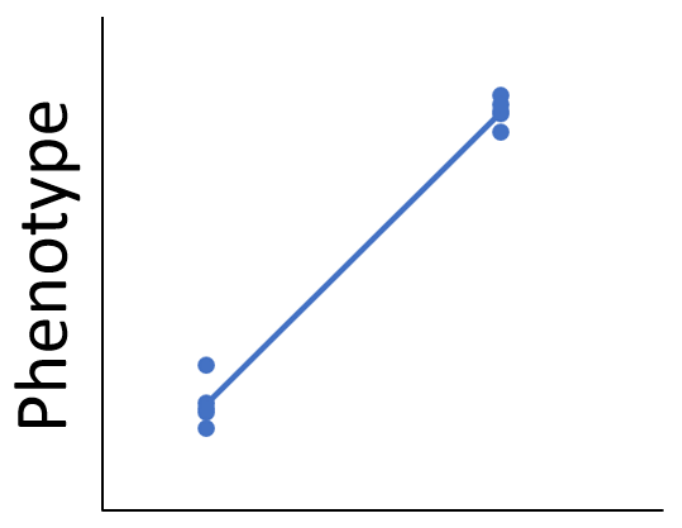

\section{Environment}

Figure 2: Example reaction norm where the phenotypes of multiple organisms (each dot) are averaged within each environment to form a reaction norm. For a real example of such a figure, see Levis, SerratoCapuchina, and Pfennig (2017, 1718).

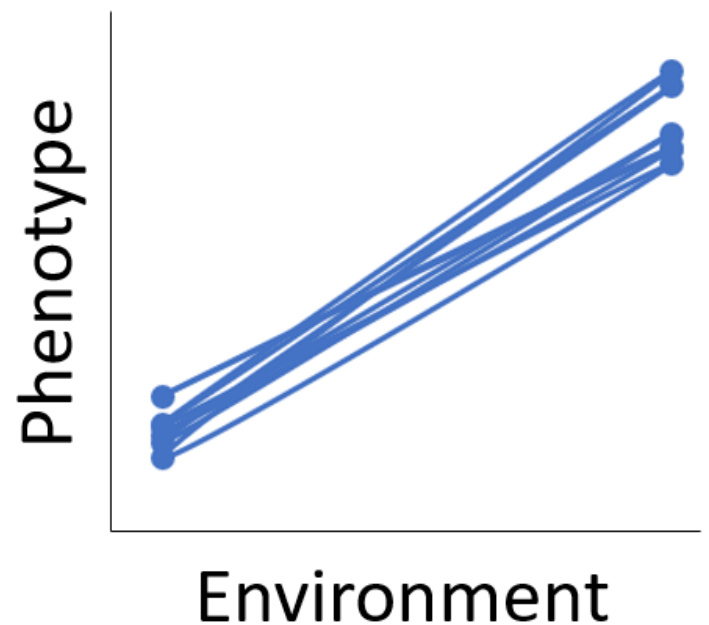

Figure 3: Example reaction norm where each organism (each dot) is paired with a "similar" organism in the other environmental condition, resulting in many reaction norm lines. For a real example of such a figure, see Gomez-Mestre et al. (2008, 796).

one genotype, without limiting us to single organisms. ${ }^{5}$ Studies of plasticity in plants (and also microorganisms) are more likely than studies of animals to be able to use clones, and thereby to be more rigorous in this respect.

Researchers who are studying irreversible plasticity in genetically heterogeneous populations currently do not distinguish their work from the work of researchers studying reversible plasticity and/or using genetically identical individuals. ${ }^{6}$ Instead, I have suggested that both of these factors - reversibility of the plasticity and genetic similarity of the test subjects—should be taken into consideration when evaluating the strength of evidence these studies provide for

5. Note that use of clones or asexually reproducing species in general is often important in studies that seek to relate particular traits to a particular genotype, including outside the context of plasticity research.

6. For some other considerations concerning organism versus population-level plasticity research, see Fuller (2003). 
the Plasticity Hypothesis. Table 1 gives some examples of studies that would be done in each of the four categories created by combining considerations of reversibility and genetic similarity. I have also included a very rough rule of thumb for which types of studies provide greater evidential support than others. Our confidence should be highest in cases where reversible plasticity is measured for genetically identical organisms. I've assigned a medium confidence level (in no particular order) to experiments where reversible plasticity is tested on conspecifics or where irreversible plasticity is tested on clones. At the lowest confidence level are experiments that test irreversible plasticity on conspecifics. Note that I have not taken seriously the suggestion to use single organisms, due to considerations of sample size. Indeed, other considerations like sample size or methodology could outweigh the confidence levels I suggest here. Certainly, some studies within each category will also be better than others.

\begin{tabular}{lll}
\hline $\begin{array}{l}\text { Confi- } \\
\text { dence }\end{array}$ & Experiment Type & Examples \\
\hline High & $\begin{array}{l}\text { Reversible plasticity; asexual reproduction } \\
\text { (clones) }\end{array}$ & $\begin{array}{l}\text { Signaling behavior in single-celled } \\
\text { organisms } \\
\text { Medium }\end{array}$ \\
$\begin{array}{l}\text { Irreversible plasticity; asexual reproduction } \\
\text { (clones) }\end{array}$ & $\begin{array}{l}\text { colonies } \\
\text { Medium }\end{array}$ \\
Reversible plasticity; sexual reproduction \\
Low & $\begin{array}{l}\text { Prey's behavioral response to } \\
\text { (conspedifics) } \\
\text { (conspecifics) }\end{array}$ & $\begin{array}{l}\text { Presence or absence of a } \\
\text { developmental trait }\end{array}$ \\
\hline
\end{tabular}

Table 1: Types of phenotypic plasticity studies, categorized by whether reversible/irreversible plasticity is being studied on genetically identical/nonidentical organisms.

I am not suggesting that experimenters have much of a choice between these different types of studies; if, in testing the Plasticity Hypothesis, what one is studying is irreversible plasticity in a sexually reproducing species—as many are-then the experiment is automatically relegated to the lowest level on my proposed evidential hierarchy. Rather, I'm suggesting that a higher or lower credence in a particular instance of the Plasticity Hypothesis is due in some types of plasticity studies over others. Evidence of reversible plasticity in genetically identical organisms should be more compelling than evidence of irreversible plasticity in sexually reproducing organisms.

Although it is outside the scope of this paper to develop this suggestion in more detail here, I am optimistic that statistical methods which take these differences in experimental design into account could satisfy my recommendation. The most obvious way that this could be done is to use (known or expected) degrees of relatedness between conspecifics in sexually reproducing species to adjust researchers' claims about the evidence for plasticity. For example, studies which use siblings which all share one parent are expected to share one quarter of their genes. Presumably, this information could be incorporated into the quantitative methods which, for instance, generate error bars on a reaction norm graph. Although it is more challenging to say exactly how to quantify the relationship between studies of reversible and irreversible plasticity, I hope that future researchers will explore this possibility in order to develop a method for rigorously distinguishing studies of various experimental designs.

Fortunately, a lot of work is being done on identifying the mechanisms of phenotypic plasticity in certain cases. I readily admit that understanding the mechanisms by which an organism ends up with one or another phenotype can help mitigate the concerns I've raised. If we un- 
derstand exactly how an organism is sensing environmental information, and converting that information into a change in phenotype, then there is no need for a reaction norm as evidence that plasticity has occurred. For example, various hormone pathways and DNA methylation have often been implicated in regulating plastic responses to environmental input (e.g., Foret et al. 2011). Studies that focus on identifying a mechanistic understanding of the plasticity certainly supplement our evidence in favor of the Plasticity Hypothesis. However, studies that identify mechanisms for plasticity are not themselves seeking evidence for the Plasticity Hypothesis. In other words, mechanism researchers are not trying to establish whether there is a (sloped) reaction norm for a particular set of traits in some environments; they are trying to establish how such a reaction norm exists. Nonetheless, establishing how plasticity is occurring does necessarily demonstrate that it is occurring, so I do not wish to rule out the possibility of using studies of mechanisms to support the Plasticity Hypothesis. Additional types of studies may provide further support as well; for instance, Kovaka (2017) argues for the use of formal modeling and experimental evolution in developmental plasticity research.

In summary, with respect to plasticity research: (1) Reaction norms, which have one line per genotype, do not perfectly test the plasticity of individual organisms. (2) Studies of irreversible plasticity rely on a counterfactual about how particular organisms would have had a phenotype in a given environment. This counterfactual cannot be tested other than by using the proxy of a genetically similar population, as of conspecifics or clones. (3) If studies use populations of conspecifics rather than clones, they are mis-attributing a property of the genotype-its reaction norm - to a population containing some genetic variation. Studies that use clones are preferable insofar as they limit this genetic variation. These considerations have helped to generate the hierarchy in table 1 .

I now turn to problems with testing whether plasticity is adaptive. As noted above, not all plasticity is adaptive. However, many researchers take special interest in phenotypic plasticity that is adaptive.

\section{Adaptive Plasticity}

As noted in the introduction, phenotypic plasticity — and especially, adaptive plasticity - has implications for evolutionary biology. The standard evolutionary story would have it that all phenotypic variation corresponds with genotypic variation. Selection acts on phenotypes, not on genotypes (because it is the phenotype that affects fitness), and so genotypes are only selected for insofar as they produce phenotypes that contribute to improving differential fitness. If the environment can also affect phenotypes, sometimes it won't be the effect of genes that is being selected for. In light of these implications, some researchers have sought to show not only that particular instances of phenotypic variation are the result of plasticity, but also that such plasticity is adaptive.

Researchers have also shown interest in the role that plasticity can play in the eventual emergence of a non-plastic, adaptive phenotype. For example, Levis and Pfennig (2016) have written about the so-called 'plasticity-first' hypothesis, which says that the emergence of a particular adaptive phenotype was facilitated by plasticity between that phenotype and the original, ancestral phenotype, after which the adaptive phenotype becomes genetically accommodated and the plasticity removed. I consider this an important topic of research (see also West-Eberhard 1989;

7. Love (2010), however, argues that the ways in which we study these mechanisms, namely the use of "model organisms," are in tension with the phenomenon of plasticity itself, since the very idea of a "model organism" assumes phenotypic stability. 
Pigliucci and Murren 2003). 8 However, I will instead focus on much simpler hypotheses: first, that observed plasticity is in fact (currently) adaptive (section 3.1), and then that the plasticity emerged as an adaptation (section 3.2).

\subsection{Adaptiveness}

This section will address tests of the following class of hypotheses:

Adaptiveness Hypothesis: A particular instance of observed phenotypic plasticity is adaptive.

Each instance of the Adaptiveness Hypothesis is not about whether plasticity overall is adaptive but about whether the plasticity of a given trait is adaptive. Any given test of the Adaptiveness Hypothesis must thus also show that the corresponding Plasticity Hypothesis from section 2 is true. Note that the Adaptiveness Hypothesis does not say that the plasticity emerged as an adaptation; I will address this further hypothesis in section 3.2. 1992):

The following are all necessary conditions to show that plasticity is adaptive (based on Moran

1. There are fitness tradeoffs 9 between the range of phenotypes.

2. The benefits of plasticity outweigh the costs.

3. There is environmental heterogeneity (over space or time).

4. The organism can perceive a relevant environmental cue.

Although there is (near) consensus among researchers that these four conditions are necessary to show that plasticity is adaptive, insufficient attention has been paid to explicating and justifying each of them. Let's take them each in turn.

1. There is a "fitness tradeoff" if and only if the following is true: for some environmental conditions and some phenotypes, some phenotypes have higher associated fitness in some environments whereas other phenotypes have higher fitness in other environments. Adaptive plasticity will be between this range of phenotypes in these environments. This is roughly what "phenotype-environment matching" refers to: using the "right" (higher fitness) phenotype in the corresponding environment. Take an easy case of two phenotypes in two environments. A fitness tradeoff will occur when Phenotype 1 has a higher fitness in Environment 1 than Phenotype 2 has in Environment 1, and Phenotype 2 has a higher fitness in Environment 2 than Phenotype 1 has in Environment 2 (or vice versa-the important thing is that each of the phenotypes have the higher fitness in one of the environments). If there is no fitness tradeoff, then one of the two phenotypes will have a higher fitness than the other regardless of the environment. In this case, it would be better for the organism in question to always exhibit the phenotype with the higher fitness, i.e., to be a "specialist" in that phenotype, rather than to exhibit plasticity between that phenotype and another, lower-fitness one. This is why it is necessary to show that there is a fitness tradeoff to show that adaptive plasticity is occurring: without a fitness tradeoff, the plasticity is maladaptive compared to specializing in one, non-plastic phenotype.

8. Although see Kovaka (2017) for problems with underdetermination in testing this hypothesis.

9. I will be using the word "tradeoff" in a technical sense distinct from the point (number 2) that the benefits of plasticity outweigh the costs. 


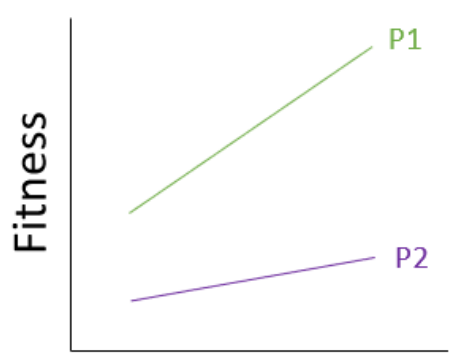

a. Environment
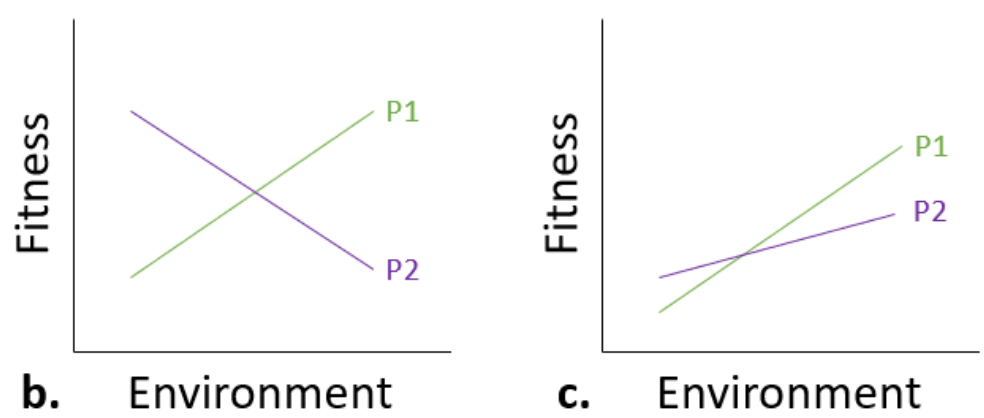

Figure 4: Example fitness functions. Only (b) and (c) show a fitness tradeoff.

Another type of graph is useful here: the fitness function. Unfortunately, fitness functions look very similar to reaction norms. However, fitness functions compare the fitness ( $y$-axis) of different phenotypes (each line) - not, as in the case of reaction norms, genotypes-in different environments ( $x$-axis). Figure 4 gives some examples. In figure $4(\mathrm{a})$, phenotype $1(P 1)$ has a higher fitness than phenotype $2(P 2)$ no matter the environment. It would be better to be a $P 1$ specialist than to be plastic between $P 1$ and $P 2$, so such plasticity would not be adaptive. Figures $4(\mathrm{~b})$ and (c) both show fitness tradeoffs. Say we are only dealing with two environments, $E 1$ (on the left of the graph) and $E 2$ (on the right). In $4(\mathrm{~b})$, the fitness of $P 1$ in $E 2$ is higher than it is in $E 1$, and the fitness of $P 2$ is higher in $E 1$ than it is in $E 2$. The fact that there is a tradeoff, however, comes from the fact that the fitness of $P 1$ in $E 1$ is less than that of $P 2$ in $E 1$, and the fitness of $P 2$ in $E 2$ is less than that of $P 1$ in $E 2$. In 4 (c), both $P 1$ and $P 2$ experience higher fitness in $E 2$ than they do in $E 1$. But there is still a tradeoff, because the fitness of $P 2$ in $E 1$ exceeds the fitness of $P 1$ in $E 1$ and the fitness of $P 1$ in $E 2$ exceeds the fitness of $P 2$ in $E 2$. For condition 1 to be met, a fitness tradeoff between the phenotypes in each environment must occur. Of course, using fitness functions with only two phenotypes and two environments is an unrealistic oversimplification, and researchers have examined adaptive plasticity in a larger number of environmental conditions. For example, in a discussion of invasive plant species, Richards et al. (2006) evaluate the relative advantages of a "jack of all trades" strategy (where an invasive species exhibits plasticity using many phenotypes in many environments) compared to a "master of some" strategy (where the species exhibits plasticity between only a few phenotypes, some of which are very advantageous in a few environments).

2. There are many potential costs to plasticity. Maintaining the sensory mechanisms necessary to detect the environment, for example, may be expensive. Clearly there will be circumstances in which the costs of plasticity outweigh the benefits. 10 Dewitt, Sih, and Wilson (1998, 78) give five possible costs of plasticity - maintenance, production, information acquisition, developmental instability, and genetic costs, including linkage, pleiotropy, and epistasis—and four possible limits to the benefits of plasticity-information reliability, lag-time, developmental range, and the epiphenotype problem. One additional candidate for a population-level cost of plasticity is that only so many alternative phenotypes can be maintained by selection without risk of being modified by mutation or drift, because only the expressed phenotypes are selected for (Snell-Rood et al. 2010). However, there is some debate about how important these costs are relative to the benefits. For example, Van Kleunen and Fischer (2005) attribute the relative lack of evidence for adaptive plasticity in plants to its high costs. On the contrary, Murren

10. Note that these costs are distinct from the costs of the phenotypes themselves; here, the question is whether there are costs involved in the plastic mechanisms themselves. 
et al. (2015) conclude that, while there must be limits to plasticity, "numerous experimental studies have not detected widespread costs" (293).

3. Environmental heterogeneity is important because if organisms do not actually experience the environments that induce the plasticity, the plasticity cannot be beneficial to fitness (see, e.g., Pigliucci 2001). Imagine that Species $A$ changes color when exposed to extremely cold temperatures, but that in its natural habitat it is never exposed to temperatures low enough and so never displays the alternate color phenotype. This phenotype would never have had the chance to confer a fitness benefit, and so the plasticity regarding color would also not have been able to confer such a benefit. One way that environmental heterogeneity may be achieved is through migration between two (or more) distinct environments (e.g., Sultan and Spencer 2002; Kawecki and Ebert 2004), but it could also happen in one location as the result of, for example, seasonal changes. The degree of environmental heterogeneity can also influence the costs and benefits of plasticity, such that plasticity will generally be more beneficial than costly when organisms are exposed to extreme environmental heterogeneity (Van Tienderen 1991). Note that it is rather difficult to show that a species never is exposed to a particular environment. So the onus is on the researchers to show that there was or is environmental heterogeneity in order to support the Adaptiveness Hypothesis. This condition also means that the Plasticity Hypothesis must, in this case, be relative to environments actually experienced by the studied organisms. There was no such constraint on the Plasticity Hypothesis outside the context of adaptiveness.

4. Recall that in West-Eberhard's definition, the organism must be able to react to an environmental input. If the organism lacks the sensory mechanisms to be able to perceive which environment it is in, it will be unable to have adaptive plasticity. The cues must be relevant because it is not enough that the organism be capable of reacting to some environmental input. The input has to at least correlate with the aspect of the environment that is relevant to fitness. Note that the cue need not be direct. For example, some tadpoles sense chemical cues, such as stress hormones, emitted by conspecifics who have been preyed on, and respond thus to the presence of a predator (e.g., Middlemis-Maher, E., and Denver 2013). They are not directly sensing the predator, but they are sensing a cue relevant to or correlated with the predator's presence.

Conditions 11 and 2 amount to saying that there must be a fitness differential between the plastic and nonplastic options: that the mean 11 fitness of plastic individuals is greater than the mean fitness of nonplastic individuals of the same phenotypes, or "specialists." (Specialists would correspond to a flat reaction norm that shared the same phenotype with the plastic genotype in one environment, as with $G 2$ in figure 1(c).) Conditions 3 and 4 are necessary to show that the potentially adaptive plasticity has a chance of actually occurring in the contexts which would make it confer this fitness benefit.

Demonstrating a fitness tradeoff can be particularly challenging. In fact, some researchers fail to show a fitness tradeoff at all, yet claim they have demonstrated an instance of adaptive plasticity. For example, Aubret, Shine, and Bonnet (2004) showed that being fed larger prey caused tiger snakes (Notechis scutatus) to grow larger jaws, whether or not they were from a mainland population that normally fed on smaller prey (and had smaller jaws) or from an island population that normally fed on larger prey (and had larger jaws). However, the researchers do not show that having a smaller jaw has higher fitness than having a larger jaw in any experimentedwith environmental condition. Thus no fitness tradeoff has been demonstrated. Nonetheless,

11. Note that the arithmetic mean should be used for spatial environmental variation while the geometric mean should be used for temporal variation (Moran 1992). This is because the effects of temporal variation have the potential to be much greater — dying out during times of environmental mismatch — so the effects are multiplicative.

๑ OPEN ACCESS - PTPBIO.ORG 
the article concludes, "Tiger snakes are highly flexible predators that track prey resources by means of a complex adaptive response" (261, emphasis added).

Other researchers argue that failing to experimentally show a fitness tradeoff doesn't necessarily show lack of adaptiveness. For example, Prudic et al. (2015) measured fitness for two morphs of Bicyclus anynana butterflies in the presence or absence of a predator (4). The wet season morph (bright eyespots) had higher longevity in the condition with a predator (a mantid) than the dry season morph (dull eyespots), and the dry season morph had higher longevity in the condition without any predator, so there was a tradeoff in terms of longevity ${ }^{12}$ However, on another fitness measure - average number of eggs laid — no tradeoff was measured; both morphs laid more eggs in the no-predator condition, and the wet season morph laid more eggs than the dry season morph in both cases. To explain this, researchers note that their two environmentswith and without mantids-do not match the two seasonal predatory environments $B$. anynana faces (2). The dry season morph butterflies are more likely to encounter vertebrate predators, such as birds, and other experiments already showed that having dull eyespots is advantageous around these predators by delaying detection (Lyytinen et al. 2004, cited by Prudic et al. 2015). So here the argument is that these two studies combined give good reason to believe there is a fitness tradeoff, although no individual study comparing vertebrate with invertebrate predation has shown this is true. Assuming that the data from these two experiments could be seamlessly integrated into a single fitness function, one which did show a fitness tradeoff, then the researchers' claim of adaptive plasticity would be justified. However, various differences between two studies may make it the case that a single fitness function is difficult to form.

As was argued in section 2 in the context of the Plasticity Hypothesis, there is thus a sense in which failure to confirm the Adaptiveness Hypothesis-in this case, failure to show a fitness tradeoff-does not disconfirm the hypothesis. Researchers can argue that their environmental conditions don't reflect the actual environmental conditions faced by the organisms being studied.13 In the case of Prudic et al. (2015), another study using different (complementary) environmental conditions had already been performed. But in principle, the argument "we did not find a fitness tradeoff; therefore, we must have contrasted unrealistic or incomplete environmental conditions" could be applied to any study that failed to measure a fitness tradeoff. This shows that if one thinks that scientific hypotheses need to be disconfirmable, the Adaptiveness Hypothesis may not be a good scientific hypothesis.

There are other lessons here that mirror those from section 2. First of all, what exactly does it mean to test the same phenotype in different environments? Researchers in the B. anyana study identify the eyespots as the relevant phenotype, and select those that have or do not have that phenotype to comprise each line on the fitness function graph. However, if one thought that "phenotype" referred more holistically to the properties of an individual organism, there is a sense in which testing multiple butterflies with the same wet or dry season morph does not exactly test multiple butterflies with the same phenotype. ${ }^{14}$ Thus, how one thinks about "the same phenotype" matters for whether one thinks that "the same phenotype" can be tested in multiple environments, at least in cases of irreversible plasticity.

12. The explanation offered is that the eyespots deflect attacks to the wings rather than the more-fatal head, thorax, or abdomen.

13. Note that the problem of matching experimental conditions to real environmental conditions applies to nearly all experimental evidence for evolutionary hypotheses, including outside of the context of phenotypic plasticity.

14. The Prudic et al. (2015) study actually had an ingenious experimental design to show that it was indeed the eye spots conferring the fitness benefit: they (gently!) glued eye spots onto the dry season morph, and covered up the naturally-occurring eye spots on the wet season morph. However, I take it the point still stands: testing different individual organisms necessarily means testing different phenotypes, to some extent. 
Second, for organisms with reversible plasticity, it may actually be impossible to put an organism with $P 1$ in $E 2$, if $E 2$ induces $P 2$ rather than $P 1$ (Schmitt, Dudley, and Pigliucci 1999). Inability to expose $P 1$ to $E 2$ while keeping the genotype constant may cause experimenters to use a proxy: a similar, specialist genotype that is unable to produce more than one phenotype regardless of the environment. (Note that this is the inverse of the problem in section 2, in which it was harder to test irreversible plasticity than reversible plasticity.) For example, Baldwin et al. (2006) studied signaling behavior in plants in response to herbivore attacks. They used genetically modified "deaf" and "mute" plant species to test the fitness conferred on the nonmutant plants by their ability to receive or transmit the signal. Using these genetically modified plants may be necessary in order to compare the plastic (signaling and signal-responsive) phenotypes to the specialist ones-as in, the researchers may not have much of a choice but to use genetically modified plants in their study-but the genetically modified plants don't have the same genotypes (or phenotypes) as the unmodified ones, and so can't serve as an unproblematic comparison class.

\subsection{Adaptation}

As mentioned above, the Adaptiveness Hypothesis does not involve hypothesizing that the plasticity emerged as an adaptation. Some researchers will wish to test the Adaptation Hypothesis:

Adaptation Hypothesis: The observed phenotypic plasticity emerged historically as the result of natural selection.

Gotthard and Nylin (1995) call attention to the fact that plasticity can be adaptive, i.e., meet the four criteria above, without necessarily having originated as an adaptation. So if experimenters want to show that the plasticity in question arose as an adaptation, they must meet another criterion, something to the effect of:

5. The plasticity originated as the result of natural selection because of its positive effect on differential fitness.

As for any other trait, showing that condition 5 is met will involve reconstructing the history of the plastic trait and showing that the plasticity did not originate as the result of drift or potentially other non-selective forces $\frac{15}{15}$ For example, the poison dart frogs' toxicity mentioned above is adaptive, but is not an adaptation, because it has been co-opted from what was likely actually maladaptive originally. Of course, any claims about adaptive plasticity will have to be relative to particular traits and particular environments, as was discussed in section 2.

Not all adaptations are currently adaptive, just as not all adaptive traits emerged as adaptations. Table 2 shows the various ways in which adaptation and adaptiveness can be combined. In order to figure out whether a particular instance of plasticity is an adaptation, it is necessary to reconstruct the ancestral conditions. If an instance of plasticity is currently found in a variable environment such that the phenotypes produced in each version of the environment are the fitter phenotypes for that environment, then that plasticity is adaptive. If plasticity emerged concurrent with environmental variation such that the plasticity was adaptive at the time, then the plasticity emerged as an adaptation. However, as is the case for other traits, adaptiveness and adaptation can come apart.

15. Of course, one necessary condition for showing that natural selection has occurred in general is to show that there is heritable phenotypic variation. I take it this will be part of showing that condition 5 has been met. Certainly this is apt to cause experimental difficulties, but it is not a crucial point in the context of the examples I highlight here.

๑ OPEN ACCESS - PTPBIO.ORG 


\begin{tabular}{|c|c|c|c|}
\hline & \multicolumn{2}{|c|}{ Adaptive? } \\
\hline & & Yes & No \\
\hline 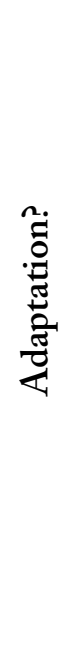 & No & $\begin{array}{l}\text { (a) After an ancestral state with } \\
\text { neither environmental variation } \\
\text { nor plasticity, plasticity emerges } \\
\text { concurrent with newly variable } \\
\text { environmental conditions. } \\
\text { Present environmental conditions } \\
\text { are variable and plasticity is } \\
\text { present. } \\
\text { (c) Ancestral state is one of } \\
\text { plasticity but no environmental } \\
\text { variation. Currently, environment } \\
\text { is variable in such a way that } \\
\text { makes preexisting plasticity } \\
\text { adaptive. }\end{array}$ & $\begin{array}{l}\text { (b) After an ancestral state with } \\
\text { neither environmental variation } \\
\text { nor plasticity, plasticity emerges } \\
\text { concurrent with newly variable } \\
\text { environmental conditions. } \\
\text { However, the present } \\
\text { environment is not variable, } \\
\text { although the plasticity persists. } \\
\text { (d) Ancestral condition is one of } \\
\text { plasticity but no environmental } \\
\text { variation. Present conditions are } \\
\text { the same. }\end{array}$ \\
\hline
\end{tabular}

Table 2: Ways in which plasticity can be adaptive, an adaptation, both, or neither. Note that "environmental variation" refers specifically to environmental variation in which the relevant trait's plasticity would be adaptive. For a graphical depiction of these categories, see Gotthard and Nylin (1995).

In table 2, cell (a), the plasticity is both adaptive and an adaptation. It evolved at the same time as the environment became heterogeneous. In cell (b), the plasticity did originate as an adaptation, but it is no longer adaptive. Like in (a), the plasticity evolved in conjunction with environmental heterogeneity, but now the environment is homogeneous. The plasticity may be fitness-neutral or maladaptive because, for example, there may be a net cost to maintaining the plastic mechanisms, which would violate condition 2. In cell (c), the plasticity emerged prior to environmental heterogeneity, so is currently adaptive, but did not emerge as an adaptation. In cell (d), the plasticity is neither adaptive nor an adaptation, because it emerged in the absence of environmental heterogeneity and still exists in that state. Note that although the case in cell (c) is of plasticity that is an adaptation without being adaptive, when the plasticity evolved, it was adaptive. At that earlier time, conditions $1-4$ would have been met.

Table 2 includes claims about the ancestral states of the environment and plasticity. In practice, these claims will be based on the current states plus hypothesized phylogenetic relationships, although certainly these inferences can be mistaken. For example, we might think that an instance of plasticity emerged as an adaptation because a taxon which we are using as a proxy for the ancestral condition does not currently display plasticity. However, it is possible that this taxon is a bad proxy for ancestral condition, having lost its plasticity over time. Additionally, our verdict that this plasticity emerged as an adaptation will depend on uncertain information about the history of the environment. Our inferences about the emergence of plasticity will also depend on our certainty in phylogenetic relationships. In table 2, these relationships are taken as a given, but in practice such relationships can be very difficult to infer. Our confidence in these relationships exists on a spectrum, in the case of plasticity as elsewhere.

For example, Kulkarni et al. (2011) studied plasticity in growth rate during the larval period for three related toad species: Pelobates cultripes, Spea multiplicata, and Scaphiopus couchii. Pelobates is the more plastic of these three species. Kulkarni et al. assumes that Pelobates, the Old World variety, also most closely approximates the ancestral condition of all three species, citing Gomez-Mestre and Buchholz (2006). Gomez-Mestre and Buchholz (2006) used a method 
called a "phylogenetic generalized least-squares model" (PGLS) to estimate the ancestral state (19024). PGLS requires a known phylogeny, which Gomez-Mestre and Buchholz in turn cite from Garcia-Paris, Buchholz, and Parra-Olea (2003). The phylogentic relationships hypothesized in Garcia-Paris, Buchholz, and Parra-Olea (2003) are the result of analyzing two mitochondrial genes and using the Maximum Likelihood method to form a tree. Which methods should be used to construct phylogenetic trees is a highly controversial question, and although Maximum Likelihood is a popular method it is far from settled on as the best or only one 16 In any case, the point is that the conclusions Kulkarni et al. draw from these phylogenetic relationships about the evolution of plasticity can only be as certain as the phylogenetic relationships themselves. This is not a problem specific to plasticity research, of course, but one that I do think tends to be underemphasized in contexts where the main object of study is plasticity rather than phylogenetics.

Other phylogenetic relationships are more clear because we have direct documentation of a species' history. Moczek and Nijhout (2003) studied dung beetles (Onthophagus taurus), for which males will either grow horns or not depending on how much nutrients they were provided with as larvae. Above a certain threshold, the beetles will grow horns. Moczek and Nijhout demonstrated that the exact threshold differs between populations from Western Australia, North Carolina, and the Mediterranean. O. taurus is native to the Mediterranean, and, we know, was introduced to the Eastern U.S. in the 1960s and to Australia between 1969 and 1983. So there is very little uncertainty about the phylogenetic relationships between these populations. Moczek and Nijhout also claim that the different thresholds were not the result of founder effects, because the thresholds of the American and Australian populations were outside the range of the native Mediterranean population. (Founder effects would have produced a threshold in the derived populations fully within the range of the ancestral population.) Precise knowledge of the species' history and the ability to rule out founder effects makes Moczek and Nijhout's study more closely show that condition 5 occurred than Kulkarni et al.'s.

Here's a final example that I particularly like: Stoks et al. (2016) studied relatively recent changes to several phenotypic traits (e.g., size at maturity, fecundity, growth rate) of Daphnia magna, a water flea. Various traits, such as size, can help protect D. magna from predatory fish. D. magna reproduces asexually most of the time (mothers beget clone daughters), but are facultative sexual reproducers, meaning they can sexually reproduce. Females can and sometimes do produce male offspring, and then mate with them. The resulting fertilized eggs then sink to the bottom of lakes where the fleas live, and go through diapause, an extended period of no development. Hatching these eggs and then allowing them to reproduce asexually enables "resurrection experiments" with large sample sizes. The clonal populations can be tested in different environments (in this case, with and without fish chemical cues). Recall that using clones was one of the ways suggested in section 2 to improve our confidence in an experiment's results. Furthermore, collecting the eggs from lake-bottom sediment cores enables experimenters such as Stoks et al. to estimate the time period from which the eggs were dropped. Stoks et al. then correlated the eggs' time periods with known changes in predation pressure (population sizes of fish). The sampled eggs were categorized into three time periods: pre-fish (1970-72), high fish (1976-79), and less fish (1988-90). The phenotypic plasticity of each of these sub-populations of $D$. magna was then studied. This enabled researchers to show a change over time in plasticity. Stoks et al. used previous studies to predict the extent to which plasticity would be more or less adaptive. Showing that the emergence of more plasticity in a trait lines up with the onset of an environment in which it is adaptive helps to show that the plasticity emerged as an adaptation.

16. Although it is outside the scope of this paper to discuss phylogenetic methods at length, see Sober (1988b).

๑ OPEN ACCESS - PTPBIO.ORG 
Of course, studies can also be done that involve artificial selection. These studies aren't useful for showing that the Adaptation Hypothesis is true, since the Adaptation Hypothesis is about natural, not artificial, selection. However, these studies can be useful for other reasons. For example, Suzuki and Nijhout (2006) used artificial selection to show that evolution can cause changes in degree of plasticity and in the relevant trait-inducing threshold. These "proof of concept" studies are important, but don't directly address the Adaptation Hypothesis (or the Adaptiveness Hypothesis).

Let's summarize: With respect to the Adaptiveness Hypothesis, several problems arise that roughly mirror those pertaining to the Plasticity Hypothesis. (1) One line per phenotype in fitness function graphs can be problematic depending on what we mean by "the same phenotype." (2) Reversible plasticity may make it impossible to observe one phenotype in an environment which induces a different phenotype. (3) If, in order to avoid (2), experimenters use a different, non-plastic (specialist) population as a proxy for what the fitness of the plastic population would be with a phenotype that does not "match" the environment, then it is unclear how well the results of the proxy species will apply to the plastic species. With respect to the Adaptation Hypothesis-which is notably distinct from the Adaptiveness Hypothesis-another problem arises: (4) We will know to varying degrees of certainty in different cases what a species' phylogenetic history has been. Making any claim about the emergence of a trait (in this case, plasticity) requires making these historical claims.

\section{Conclusion}

The Plasticity Hypothesis is a hypothesis about the existence of a trait-plasticity-which is hypothesized to cause certain observed phenotypic variations. The Adaptiveness Hypothesis is about some features of that trait: namely, showing that the trait confers a net fitness benefit on the organisms which possess it. The Adaptation Hypothesis is a hypothesis about the history of that trait, rather than the trait's existence or its qualities.

The three lessons from section 2, regarding the Plasticity Hypothesis, are somewhat analogous to the first three lessons from section 3, regarding the Adaptiveness Hypothesis. The fourth lesson from section 3, regarding the Adaptation Hypothesis, is separate. These results, which cumulatively make up the main claims of this paper, are summarized in table B. Many of these concerns, such as those related to genetic similarity between organisms in a study, or uncertainty about phylogenetic relationships, should be familiar to those who work on evolutionary hypotheses in general. However, the sections above clarify the connection between these general issues in evolutionary theory and empirical research on plasticity. I hope that making these challenges explicit will be helpful for future experimenters and theorists alike.

It is crucial that all of the conditions given for successful testing of the Plasticity, Adaptiveness, and Adaptation hypotheses are necessary, not sufficient, conditions. It is in general extremely difficult to say what sufficient conditions would be for definitively confirming a scientific hypothesis. These necessary conditions can also be met to varying degrees; much of the preceding discussion has been about which types of experiments are better or worse than others, on a spectrum. Although it was outside of the scope of this paper to address this possibility in full, other plasticity-related studies (such as simulations, or studies which establish the mechanisms for phenotypic plasticity) could help to fill in these gaps. The result would be something like the "methodological omnivory" advocated for by Currie (2015).

All of the important implications of plasticity research-its effect on the nature versus nurture debate, and on evolutionary theory - rely on claims that plasticity is sometimes occurring, that it can be adaptive, and that it can emerge as an adaptation. This paper has pointed out the

๖ OPEN ACCESS - PTPBIO.ORG 


\begin{tabular}{lll}
\hline Plasticity Hypothesis & Adaptiveness Hypothesis & Adaptation Hypothesis \\
\hline $\begin{array}{l}\text { 1. Testing for plasticity of one } \\
\text { genotype does not test } \\
\text { plasticity for one organism }\end{array}$ & $\begin{array}{l}\text { 1. Testing for fitness of } \\
\text { phenotypically similar groups of } \\
\text { organisms in similar environments } \\
\text { does not test the fitness of one } \\
\text { phenotype in one environment. }\end{array}$ & \\
$\begin{array}{l}\text { 2. For irreversible plasticity, it } \\
\text { is impossible to observe the } \\
\text { phenotype an organism would } \\
\text { have had in a different } \\
\text { environment. }\end{array}$ & $\begin{array}{l}\text { impossible to observe a phenotype in } \\
\text { the environment which does not } \\
\text { induce it. }\end{array}$ & \\
$\begin{array}{l}\text { 3. Populations of conspecifics } \\
\text { can only approximate a test for } \\
\text { plasticity of a genotype. }\end{array}$ & $\begin{array}{l}\text { 3. Non-plastic organisms which are } \\
\text { only closely related to plastic } \\
\text { organisms can only approximate tests } \\
\text { for the plastic organisms' fitness. }\end{array}$ & \\
& & $\begin{array}{l}\text { 4. Uncertainty about a } \\
\text { species' phylogenetic }\end{array}$ \\
& & $\begin{array}{l}\text { history can effect claims } \\
\text { about the emergence of its } \\
\text { (plastic) traits. }\end{array}$ \\
\hline
\end{tabular}

Table 3: Summary of claims from previous sections.

ways in which experimentally confirming these three hypotheses can be challenging, and has suggested that some experimental contexts are better equipped than others to deal with claims about phenotypic plasticity.

\section{Acknowledgments}

The author wishes to thank Russell Powell, Alan Love, Victor Kumar, Rory Smead, and Federica Bocchi, as well as the anonymous reviewers, for their insightful feedback on this paper. The paper also greatly benefited from discussions with Karen Warkentin and her students. This material is based upon work supported by the National Science Foundation Graduate Research Fellowship Program under Grant No. DGE-1840990. Any opinions, findings, and conclusions or recommendations expressed in this material are those of the author and do not necessarily reflect the views of the National Science Foundation.

\section{Literature cited}

Aubret, F., R. Shine, and X. Bonnet. 2004. "Adaptive Developmental Plasticity in Snakes: Genes and Environment Stretch Snake Jaws to Meet the Demands of Prey Size.” Nature 431:261-262. https ://doi.org/10.1038/431261a.

Baldwin, I. T., R. Halitschke, A. Paschold, C. C. Von Dahl, and C. A. Preston. 2006. "Volatile Signalling in Plant-Plant Interactions: 'Talking Trees' in the Genomics Era.” Science 311:812-815. https://doi.org/10.1126/science.1118446.

Bull, J. J. 1980. “Sex Determination in Reptiles.” Quarterly Review of Biology 55:3-21. https://doi.org/ $10.1086 / 411613$. 
Currie, Adrian. 2015. "Marsupial Lions and Methodological Omnivory: Function, Success and Reconstruction in Paleobiology." Biology and Philosophy 30:187-209. https://doi.org/10.1007/s10539014-9470-y.

Dewitt, T. J., A. Sih, and D. S. Wilson. 1998. "Costs and Limits of Phenotypic Plasticity." TREE 13 (2): 77-81. https://doi.org/10.1016/s0169-5347(97)01274-3.

Emlen, D. J., and H. F. Nijhout. 1999. "Hormonal Control of Male Horn Length Dimorphism in the Horned Beetle Onthophagus Taurus." Journal of Insect Physiology 45:45-53. https://doi.org/10. 1016/s0022-1910(98)00096-1.

Foret, S., R. Kucharski, M. Pellegrini, S. Feng, S. E. Jacobsen, G. E. Robinson, and R. Maleszka. 2011. "DNA Methylation Dynamics, Metaboloc Fluxes, Gene Splicing, and Alternative Phenotypes in Honey Bees." Proceedings of the National Academy of Sciences USA 109 (13): 4968-4973. https://doi.org/10.1073/pnas.1202392109.

Fuller, T. 2003. "The Integrative Biology of Phenotypic Plasticity." Biology and Philosophy 18:381-389. https://doi.org/10.1023/a:1023948505327.

Garcia-Paris, M., D. R. Buchholz, and G. Parra-Olea. 2003. "Phylogenetic Relationships of Pelobatoidea Re-Examined Using mtDNA.” Molecular Phylogenetics and Evolution 28:12-23. https: //doi.org/10.1016/s1055-7903(03)00036-8.

Gilbert, S. F., and D. Epel. 2015. Ecological Developmental Biology: The Environmental Regulation of Development, Health, and Evolution. 2nd ed. Sunderland, MA: Sinauer Associates, Inc.

Gomez-Mestre, I., and D. R. Buchholz. 2006. "Developmental Plasticity Mirrors Differences among Taxa in Spadefoot Toads Linking Plasticity and Diversity." Proceedings of the National Academy of Sciences of the United States 103:19021-19026. https://doi.org/10.1073/pnas.0603562103.

Gomez-Mestre, I., J. C. Touchon, V. L. Saccoccio, and K. M. Warkentin. 2008. "Genetic Variation in Pathogen-Induced Early Hatching of Toad Embryos." Journal of Evolutionary Biology 21:791800. https://doi.org/10.1111/j.1420-9101.2008.01509.x.

Gotthard, K., and S. Nylin. 1995. "Adaptive Plasticity and Plasticity as an Adaptation: A Selective Review of Plasticity in Animal Morphology and Life History.” Oikos 74 (1): 3-17. https://doi. org/10.2307/3545669.

Gould, S. J., and R. C. Lewontin. 1979. "The Spandrels of San Marco and the Panglossian Paradigm: A Critique of the Adaptationist Programme." Proceedings of the Royal Society B 205 (1161): 581598. https://doi.org/10.4324/9781315161921-11.

Heil, M. 2010. "Plastic Defense Expression in Plants." Evolutionary Ecology 24:555-569. https://doi. org/10.1007/s10682-009-9348-7.

Kamakura, M. 2011. "Royalactin Induces Queen Differentiation in Honeybees.” Nature 473:478-483. https://doi.org/10.1038/nature10093.

Kawecki, Tadeusz J., and Dieter Ebert. 2004. “Conceptual Issues in Local Adaptation.” Ecology Letters 7:1225-1241. https://doi.org/10.1111/j.1461-0248.2004.00684.x.

Keller, E. F. 2010. The Mirage of a Space Between Nature and Nurture. Durham, NC: Duke University Press.

Kovaka, Karen. 2017. "Underdetermination and Evidence in the Developmental Plasticity Debate." British Journal for the Philosophy of Science 70 (1): 127-152. https://doi.org/10.1093/bjps/axx038. 
Kulkarni, S. S., I. Gomez-Mestre, C. L. Moskalik, B. L. Storz, and D. R. Buchholz. 2011. "Evolutionary Reduction of Deveopmental Plasticity in Desert Spadefoot Toads." Journal of Evolutionary Biology 24:2445-2455. https://doi.org/10.1111/j.1420-9101.2011.02370.x.

Laland, K., T. Uller, M. Feldman, K. Sterelny, G. B. Müller, A. Moczek, E. Jablonka, et al. 2014. “Does Evolutionary Theory Need a Rethink?” Nature 514 (7521): 161-164. https://doi.org/10. $1038 / 514161 \mathrm{a}$.

Levis, N. A., A. Serrato-Capuchina, and D. W. Pfennig. 2017. "Genetic Accommodation in the Wild: Evolution of Gene Expression Plasticity during Character Development.” Journal of Evolutionary Biology 30:1712-1723. https://doi.org/10.1111/jeb.13133.

Levis, Nicholas A., and David W. Pfennig. 2016. "Evaluating 'Plasticity-First' Evolution in Nature: Key Criteria and Empirical Approaches." Trends in Ecology and Evolution 31 (7): 563-574. https: //doi.org/10.1016/j.tree.2016.03.012.

Lewens, T. 2010. "What Are 'Natural Inequalities'?” The Philosophical Quarterly 60 (239): 264-285. https://doi.org/10.1093/acprof:oso/9780198712657.003.0009.

Lewontin, R. C. 2006. “The Analysis of Variance and the Analysis of Causes.” International Journal of Epidemiology 35:520-525. https://doi.org/10.1093/ije/dyl062.

Love, Alan. 2010. "Idealization in Evolutionary Developmental Investigation: A Tension between Phenotypic Plasticity and Normal Stages." Philosophical Transactions of the Royal Society B 365:679690. https://doi.org/10.1098/rstb.2009.0262.

Lyytinen, A., P. M. Brakefield, L. Lindstrom, and J. Mappes. 2004. "Does Predation Maintain Eyespot Plasticity in Bicyclus Anynana?" Proceedings of the Royal Society B 271:279-283. https://doi. org/10.1098/rspb.2003.2571.

McShea, D. W. 2012. "Upper-Directed Systems: A New Approach to Teleology in Biology." Biology and Philosophy 27:663-684. https://doi.org/10.1007/s10539-012-9326-2.

Middlemis-Maher, J., Werner E. E., and R. J. Denver. 2013. "Stress Hormones Mediate PredatorInduced Phenotypic Plasticity in Amphibian Tadpoles." Proceedings of the Royal Society B 280 (1758): 2123075. https://doi.org/10.3389/conf.fendo.2011.03.00031.

Moczek, A. P., and H. F. Nijhout. 2003. "Rapid Evolution of a Polyphenic Threshold." Evolution and Development 5 (3). https://doi.org/10.1046/j.1525-142x.2003.03033.x.

Moran, N. A. 1992. “The Evolutionary Maintenance of Alternative Phenotypes.” The American Naturalist 139 (5): 971-989. https://doi.org/10.1086/285369.

Murren, C. J., J. R. Auld, H. Callahan, C. K. Ghalambor, C. A. Handelsman, M. A. Heskel, J. G. Kingsolver, et al. 2015. "Constraints on the Evolution of Phenotypic Plasticity: Limits and Costs of Phenotypic Plasticity.” Heredity 115:293-301. https://doi.org/10.1038/hdy.2015.8.

Nijhout, H. F. 1991. The Development and Evolution of Butterfly Wing Patterns. Washington, DC: Smithsonian Institution Press.

Pigliucci, M. 2001. Phenotypic Plasticity: Beyond Nature and Nurture. Baltimore, MD: The Johns Hopkins University Press.

Pigliucci, M. 2007. "Do We Need an Extended Evolutionary Synthesis?” Evolution 61 (12): 27432749. https://doi.org/10.1111/j.1558-5646.2007.00246.x.

Pigliucci, Massimo, and Courtney J. Murren. 2003. "Perspective: Genetic Assimilation and a Possible Evolutionary Paradox: Can Macroevolution Sometimes Be So Fast as to Pass Us By?” Evolution 57 (7): 1455-1464. https://doi.org/10.1554/02-381. 
Prudic, K. L., C. Jeon, H. Cao, and A. Monteiro. 2011. "Developmental Plasticity in Sexual Roles of Butterfly Species Drives Mutual Sexual Ornamentation.” Science 331:73-76. https://doi.org/10. 1126/science.1197114.

Prudic, K. L., A. M. Stoehr, B. R. Wasik, and A. Monteiro. 2015. "Eyespots Deflect Predator Attack Increasing Fitness and Promoting the Evolution of Phenotypic Plasticity." Proceedings of the Royal Society B 282:20141531. https://doi.org/10.1098/rspb.2014.1531.

Richards, Christina L., Oliver Bossdorf, Norris Z. Muth, Jessica Gurevitch, and Massimo Pigliucci. 2006. "Jack of All Trades, Master of Some? On the Role of Phenotypic Plasticity in Plant Invasions.” Ecology Letters 9:981-993. https://doi.org/10.1111/j.1461-0248.2006.00950.x.

Schlichting, C. D., and H. Smith. 2002. "Phenotypic Plasticity: Linking Molecular Mechanisms with Evolutionary Outcomes.” Evolutionary Ecology 16:189-211. https://doi.org/10.1023/a:10196244 25971.

Schmitt, J., S. A. Dudley, and M. Pigliucci. 1999. "Manipulative Approaches to Testing Adaptive Plasticity: Phytochrome-Mediated Shade-Avoidance Responses in Plants." American Naturalist 154 (S1): S43-S54. https://doi.org/10.1086/303282.

Snell-Rood, E. C., J. D. Van Dyken, T. Cruickshank, M. J. Wade, and A. P. Moczek. 2010. "Toward a Population Genetic Framework of Developmental Evolution: The Costs, Limits, and Consequences of Phenotypic Plasticity." Bioessays 32 (1): 71-81. https:// doi . org/10.1002/bies. 200900132.

Sober, E. 1988a. “Apportioning Causal Responsibility.” The Journal of Philosophy 85 (6): 303-318. https ://doi.org/10.2307/2026721.

Sober, E. 1988b. Reconstructing the Past: Parsimony, Evolution and Inference. Cambridge, MA: MIT Press.

Stoks, R., L. Govaert, K. Pauwels, B. Jansen, and L. De Meester. 2016. "Reconstructing Complexity: The Interplay of Plasticity and Rapid Evolution in the Multiple Trait Response to Strong Changes in Predation Pressure in the Water Flea Daphnia Magna." Ecology Letters 19:180-190. https://doi.org/10.1111/ele.12551.

Sultan, Sonia E., and Hamish G. Spencer. 2002. "Metapopulation Structure Favors Plasticity over Local Adaptation.” The American Naturalist 160 (2): 271-283. https://doi.org/10.1086/341015.

Suzuki, Y., and H. F. Nijhout. 2006. "Evolution of a Polyphenism by Genetic Accommodation.” Science 311:650-652. https://doi.org/10.1126/science.1118888.

Tarvin, R. D., C. M. Borghese, W. Sachs, J. C. Santos, Y. Lu, L. A. O’Connell, D. C. Cannatella, et al. 2017. "Interacting Amino Acid Replacements Allow Poison Frogs to Evolve Epibatidine Resistance.” Science 357 (6357): 1261-1266. https://doi.org/10.3410/f.731256119.793539017.

Van Kleunen, Mark, and Markus Fischer. 2005. "Constraints on the Evolution of Adaptive Phenotypic Plasticity in Plants.” New Phytologist 166 (1): 49-60. https://doi.org/10.1111/j.1469-8137.2004. 01296.x.

Van Tienderen, Peter H. 1991. "Evolution of Generalists and Specialists in Spatially Heterogeneous Environments.” Evolution 45 (6): 1317-1331. https://doi.org/10.1111/j.1558-5646.1991. tb02638.x.

Warkentin, K. M. 2005. "How Do Embryos Assess Risk? Vibrational Cues in Predator-Induced Hatching of Red-Eyed Treefrogs." Animal Behavior 70:503-510. https://doi.org/10.1016/j. anbehav.2004.09.019. 
West-Eberhard, M. J. 1989. "Phenotypic Plasticity and the Origins of Diversity." Annual Review of Ecology and Systematics 20:249-278. https://doi.org/10.1146/annurev.es.20.110189.001341.

West-Eberhard, M. J. 2003. Developmental Plasticity and Evolution. New York, NY: Oxford University Press.

Wray, G. A., H. E. Hoekstra, D. J. Futuyama, R. E. Lenski, T. F. C. Mackay, D. Schluter, and J. E. Strassmann. 2014. "Does Evolutionary Theory Need a Rethink?” Nature 514 (7521): 161-164. https://doi.org/10.1038/514161a.

(C) 2020 Author(s)

This is an open-access article distributed under the terms of the Creative Commons Attribution 4.0 International license, which permits anyone to download, copy, distribute, display, or adapt the text without asking for permission, provided that the creator(s) are given full credit.

ISSN 2475-3025 